\title{
Assessment of Pulsed Magnetic Field Stimulus by Using Finger Photoplethysmogram and Pressure Pulse Waveform
}

\author{
Jinyong Lee, Insuk Go, Jaewon Choi, Taesun Jang, Sang Hoon Shin, \\ Hyun Sook Lee, Do Guwn Hwang, and Sunwook Kim* \\ Department of Oriental Biomedical Engineering, Sangji University, Kangwon 220-702, Korea
}

(Received 23 October 2010, Received in final form 1 December 2010, Accepted 16 December 2010)

\begin{abstract}
Photoplethysmogram (PPG) and pressure pulse waveform (PPW) were compared and evaluated for the efficacy of stimulating knuckles by using the pulsed magnetic field. Both signals were observed simultaneously while the knuckles were exposed for $10 \mathrm{~min}$ to the pulsed magnetic field, with maximum field intensity of $0.8 \mathrm{~T}$ and transition time of 0.126 msec. After 5 min stimulation of the knuckles, the results showed that the aging indexes calculated from the second derivative of the PPG were increased from -1.913 to 0.072 , and that of the PPW from -0.063 to 0.387 . However, for the relatively long-term stimulation for $10 \mathrm{~min}$, we found that the values of both the aging indexes of the second derivatives and augmentation index of the PPW returned to the starting level. The changes observed in characteristic factors such as the aging indexes of the second derivatives and augmentation index of the PPW indicate the potential of pulsed magnetic field stimulation as a therapeutic method for the treatment of patients with peripheral vascular disease.
\end{abstract}

Keywords : pulsed magnetic field stimulation, photoplethysmogram, pressure pulse waveform, the second derivative, augmentation index, aging index

\section{Introduction}

Pulsed electromagnetic fields have been used as therapeutic agents over the last 40 years, following convincing evidence that electric currents can accelerate bone formation [1]. Specifically, electromagnetic-field stimulation gained credibility as a therapy following observations that the application of physical stress on bones promoted the formation of very small electric currents that are related to bone formation. A similar mechanism has been observed for cartilage, whereby electrical stimulation of chondrocytes increased the synthesis of the major component of cartilage matrix, known as proteogylcans [2].

Recently, stimulation of the human body by using pulsed electromagnetic fields has gained interest as an alternative non-invasive medical treatment for peripheral vascular disease as well as a musculoskeletal dysfunction. Some results showed that the vascular blood flow can be increased by pulsed electromagnetic field stimulation. For example, Mayrovitz and Larsen found that exposure to pulsed magnetic field for 40 min significantly increased

*Corresponding author: Tel: +82-33-730-0413

Fax:+82-33-738-7962, e-mail: skim@sangji.ac.kr blood perfusion (by 29\%) in the exposed arm. Furthermore, they also indicated that this method was effective in increasing the blood perfusion in the peri-ulcer skin microcirculation of diabetic patients. These researchers suggested that microvessel recruitment may be responsible for the increases in perfusion and volume after exposure, since there was no change in blood velocity after exposure [3, 4].

In addition, there is much interest regarding the microcirculatory system. Microcirculation is the blood flow through the microvasculature, i.e., the arterioles, capillaries, and venules. These vessels nourish the body's tissues and organs. Two important functions of the microcirculatory system are alteration of the blood flow according to the varying metabolic requirements of the tissues it serves and stabilization of the blood flow and pressure by making local regulatory adjustments [5-8]. Accordingly, a greater understanding of this vascular network has, and probably will in the future, lead to advances in tissue regeneration, pain control, circulatory disorders, etc. In fact, several attempts have been made to explore the parameters of microcirculation and microvasculature when tissue and/or blood vessels have been exposed to pulsed magnetic field. 
With regard to the stimulation methods, an electromagnetic field with a low frequency and a weak intensity has been studied in several ways for its adjunctive use in the palliative treatment of postoperative pain and edema in superficial soft tissues and in the treatment of Parkinson disease [9]. Most of the physiological effects of electromagnetic field stimulation on cells and tissues were investigated at intensities of a few tens of milliteslas and at a frequency range of $6.4 \sim 75 \mathrm{~Hz}$ [10].

In this work, we determined the vascular stiffness related to vascular aging and the sclerosis level in blood vessels by simultaneous acquisition of the pressure pulse wave (PPW) and photoplethysmogram (PPG) under pulsed electromagnetic field stimulation of the knuckles. In order to identify correlation factors, comparison of the 2 waveforms and the second derivatives was also performed to take account for the effects of the pulsed magnetic field stimulation.

\section{Experimental Method}

The schematic diagram for the experimental setup is shown in Fig. 1. The generating coil was wound to a single layer of 10 turns with a hollow rectangle shape of $9.0 \times 1.2 \mathrm{~cm}$ using a flat square-shaped single stranded wire of $1 \mathrm{~mm}$ in thickness and $3 \mathrm{~mm}$ in width. Our system was designed to generate a pulsed magnetic field that had a maximum intensity variation of $0.8 \mathrm{~T}$ at a transition time of $0.126 \mathrm{msec}$. To stimulate the knuckles, the generating coil was placed under the fingers. To investigate the change in the blood flow in the peripheral vessel caused by the magnetic field stimulation, the PPW system PP-1000 (Hanbyul Meditech Co., Ltd., Korea) and the photoplethysmograph MP35 (Biopac Systems Inc., CA) were attached to the region of the radial artery and the middle phalanx of the middle finger of a hand, respectively. The PPW and PPG were recorded simultaneously under pulsed magnetic field stimulation for $10 \mathrm{~min}$.

\section{Results and Discussion}

For the stimulation of the knuckles, the 2 waveforms obtained from the PPG and PPW showed the same features (Figs. 2a and b). The PPW had more apparent peaks that were used to calculate the augmentation index and to assess alterations in the blood perfusion due to arterial sclerosis or vascular aging (points P1, P2, and P3 in Fig. 2a). However, the PPG measured at the end of the middle finger did not show any notable peaks (arrows in the Fig. 2b). The augmentation index is usually calculated as (P2-P3)/(P2-P1) [11, 12].

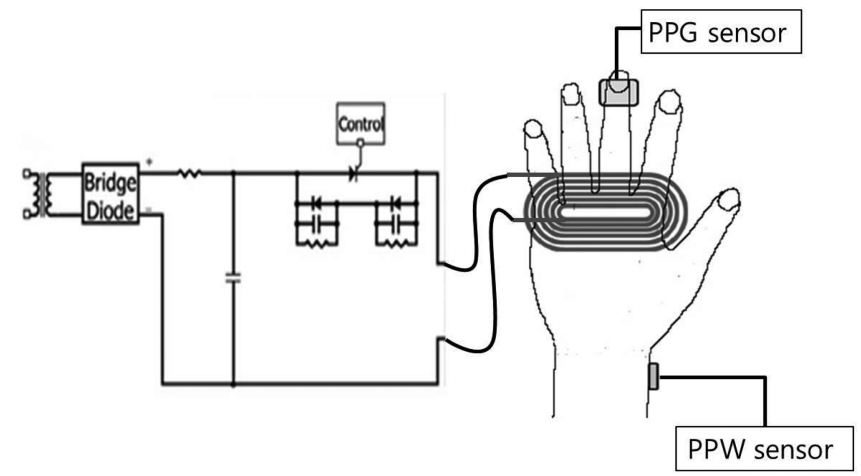

Fig. 1. Schematic diagram of the stimulation device, the photoplethysmogram, and the pressure pulse wave.

Accordingly, the augmentation indexes calculated for the different stimulation times are shown in Table 1; the values of $3 \mathrm{~min}$ and $5 \mathrm{~min}$ stimulation were increased to 0.896 (SD: 0.04) and decreased to 0.718 (0.04), respectively, compared to the starting value $0.768(0.04)$. Even if those differences were relatively small, we thought they might have been caused by the pulsed magnetic field stimulation. After stimulation for $10 \mathrm{~min}$, the value of the augmentation index returned to the starting value. Thus, we propose that this effect was caused by adaptation to the stimulus due to the homeostatic behavior of the circulation system.

The PPWs are reflected back from the periphery and are summated with the forward-going wave to produce the characteristic aortic pressure waveform. However, the second derivative of the PPG is more useful as an alternative method for the assessment of arterial elastic properties than the PPW for the evaluation of characteristics of peripheral vascular diseases such as vascular aging and sclerosis level.

The second derivatives of the 2 waveforms were calculated as shown in Figs. 2c and d, where these 2 figures were emphasized by 3 times against the time axis for the visibility. As shown in these figures, the second derivatives seem to be much clearer to evaluate the effect of the pulsed magnetic field stimulation than the original waveforms. In general, the second derivative of the PPG consists of 4 points in the systole and 1 in the diastole as noted in Fig. 2d. According to many clinical results, each index is thought to be related to the characteristics of blood perfusion such as vasodilation, vasoconstriction, and vascular compliance. Although many PPG-derived parameters are defined in terms of the ratio of the height of the waves to that of the $a$-point, we tried to focus on the aging index, which is defined as $(b-c-d-e) / a$, for assessing the effect of pulsed magnetic field stimulation $[13,14]$.

Fig. 3 and 4 show the indexes calculated from the 
(a)

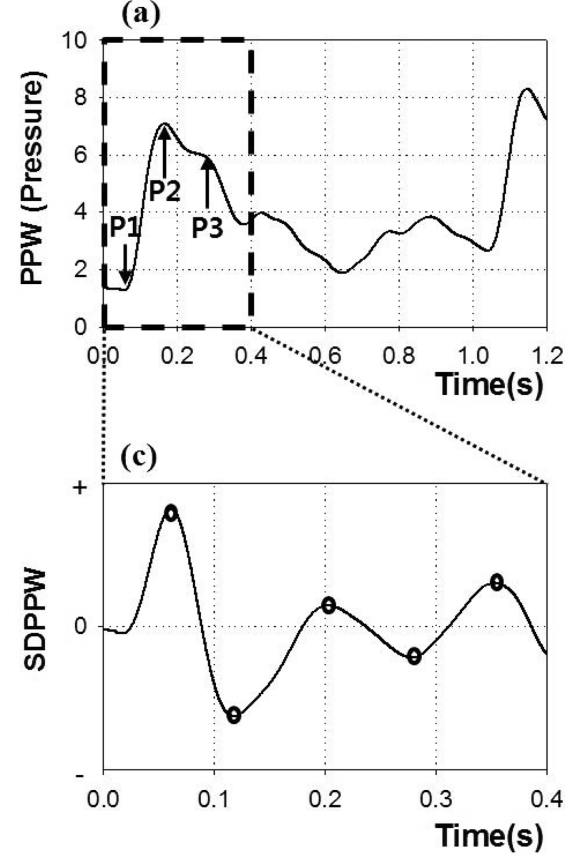

(b)
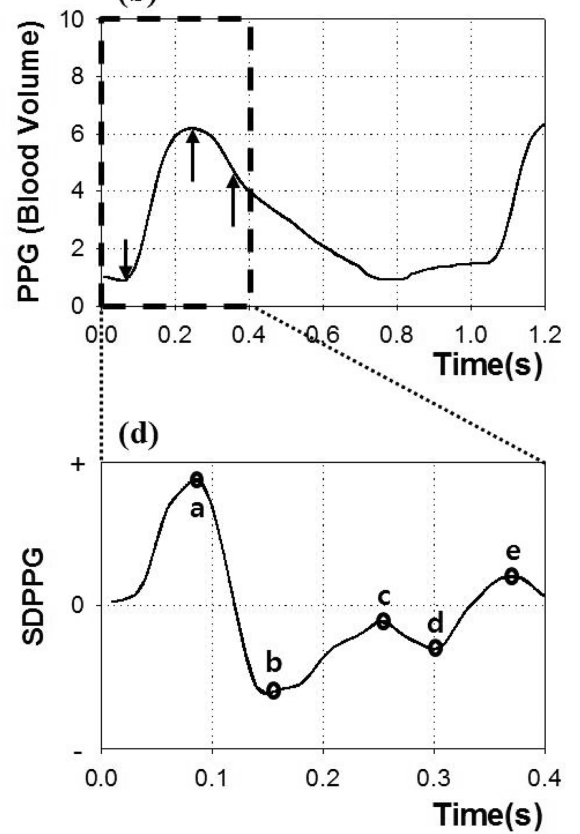

Fig. 2. (a) The pressure pulse waveform $\left(\mathrm{P}_{1}\right.$, ascending aortic diastolic pressure; $\mathrm{P}_{2}$, ascending aortic early peak systolic pressure; $\mathrm{P}_{3}$, ascending aortic late peak systolic pressure) and (b) the photoplethysmography waveform. (c) The second derivative of pressure pulse waveforms and (d) the second derivative of photoplethysmography waveform (a wave, the initial positive wave; $b$ wave, early negative wave; $\mathrm{c}$ wave, re-increasing wave; $\mathrm{d}$ wave, re-decreasing wave; and diastolic e wave). The aging index was defined as (bc-d-e)/a.

Table 1. The augmentation index calculated with stimulating time.

\begin{tabular}{cccccc}
\hline \hline & Before & $3 \min$ & $5 \min$ & $7 \min$ & $10 \min$ \\
\hline P1 & $0.130 \pm 0.37$ & $1.183 \pm 0.37$ & $-3.107 \pm 0.18$ & $1.930 \pm 0.04$ & $1.653 \pm 0.29$ \\
P2 & $6.523 \pm 0.46$ & $7.403 \pm 0.04$ & $1.340 \pm 0.10$ & $5.720 \pm 0.67$ & $4.997 \pm 0.27$ \\
P3 & $5.043 \pm 0.53$ & $6.757 \pm 0.21$ & $0.087 \pm 0.21$ & $4.787 \pm 0.90$ & $4.223 \pm 0.27$ \\
AI & $0.768 \pm 0.04$ & $0.896 \pm 0.04$ & $0.718 \pm 0.04$ & $0.754 \pm 0.10$ & $0.769 \pm 0.06$ \\
\hline
\end{tabular}

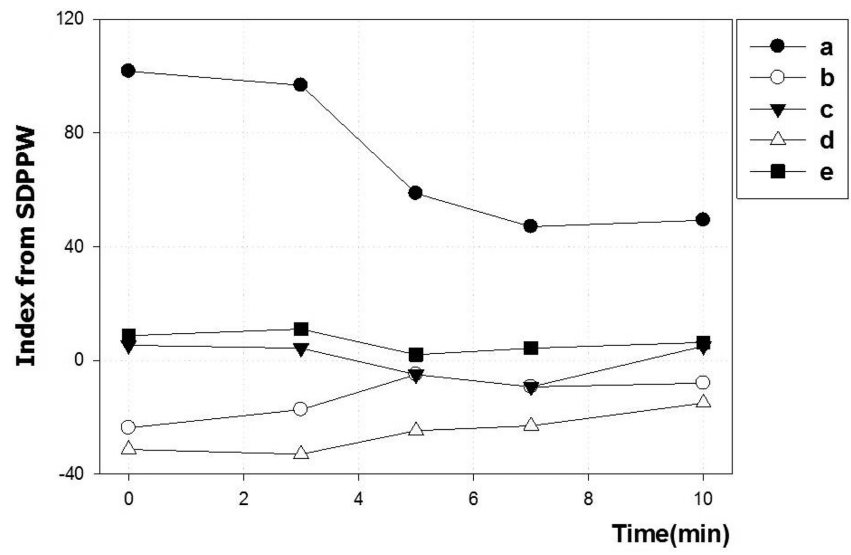

Fig. 3. The index value calculated from the second derivative of the pressure pulse waveforms (a wave, the initial positive wave; $\mathrm{b}$ wave, early negative wave; $\mathrm{c}$ wave, re-increasing wave; $d$ wave, re-decreasing wave; and diastolic e wave).

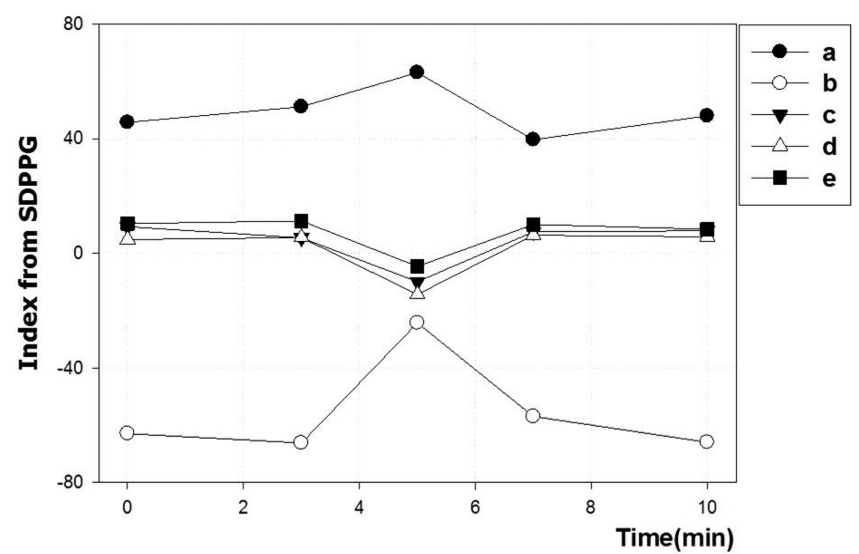

Fig. 4. The index value calculated from the second derivative of the photoplethysmogram (a wave, the initial positive wave; $\mathrm{b}$ wave, early negative wave; c wave, re-increasing wave; $d$ wave, re-decreasing wave; and diastolic e wave). 
Table 2. The aging indexes calculated from second derivative of the pressure pulse waveforms and the photoplethysmogram.

\begin{tabular}{lccccc}
\hline \hline & Before & $3 \mathrm{~min}$ & $5 \mathrm{~min}$ & $7 \mathrm{~min}$ & $10 \mathrm{~min}$ \\
\hline SDPPW & -0.063 & 0.004 & $\underline{\mathbf{0 . 3 8 7}}$ & $\underline{\mathbf{0 . 3 9 8}}$ & -0.087 \\
SDPPG & -1.913 & -1.728 & $\underline{\mathbf{0 . 0 7 2}}$ & -2.038 & -1.836 \\
\hline
\end{tabular}

second derivatives of the PPWs and the PPGs, respectively. As shown in these tables, there was no consistency between these values, except for the $5 \mathrm{~min}$ stimulation, which could be explained because we just focused on the aging index and did not evaluate other data in this study. In this study, the 2 aging indexes calculated from both waveforms have been shown to have the same tendency. The aging index calculated from the PPW was increased from -0.063 to 0.387 after stimulation for $5 \mathrm{~min}$ and then decreased to -0.087 after stimulation for $10 \mathrm{~min}$ (Tables 2 ). The aging index calculated from the PPG was also increased from -1.913 to 0.072 after stimulation for 5 min and then decreased to -1.836 after stimulation for 10 min. Unlike the patterns in the augmentation index of the PPW, the aging index increased and decreased, which was more apparent in the second derivatives. These changes in the behavior in relation to the stimulation time are thought to be caused by adaptation to the stimulus due to the homeostatic behavior of the circulation system. Consequently, the treatment time with pulsed magnetic field stimulation should be optimized with regard to age, gender, sclerosis level, etc. for its clinical application.

\section{Conclusion}

We have stimulated knuckles with pulsed magnetic fields for different stimulation times and tried to assess the effect of this treatment by using the PPW system and photoplethysmography. We found that the pulsed magnetic field may affect the blood perfusion due to changes in the peripheral vasculature noted after stimulation for 5 min, but not after stimulation for $10 \mathrm{~min}$. On the basis of these results, we suggest that analysis of the aging indexes obtained from the second derivatives of either the PPG or PPW may be useful for evaluating the efficacy of pulsed magnetic field stimulation of the knuckles of patients with diabetic peripheral vascular disease. Moreover, this assess- ment method can be utilized for optimizing the treatment time of pulsed magnetic field stimulation. In order to obtain statistical significance, clinical data acquisition should be performed according to the experimental plan approved by the institutional review board.

\section{Acknowledgment}

"This research was financially supported by the Ministry of Education, Science Technology (MEST) and Korea Institute for Advancement of Technology (KIAT) through the Human Resource Training Project for Regional Innovation.

\section{References}

[1] C. Vallbona and T. Richards, Physical Medicine and Rehabilitation Clinics of North America 10, 3 (1999).

[2] J. Hulme, V. Robinson, R. DeBie, G. Wells, M. Judd, and P. Tugwell, Cochrane Library, Oxford, 3 (2002).

[3] H. N. Mayrovitz and P. B. Larsen, Wounds 4, 197 (1992).

[4] H. N. Mayrovitz and P. B. Larsen, Wounds 7, 90 (1995).

[5] G. Kaley and B. M. Altura, Microcirculation, Univ. Park Press, Baltimore (1977) pp. 3-9.

[6] M. Neeman and H. Dafni, Annu. Rev. Biomed. Eng. 5, 29 (2003).

[7] A. Popel and P. Johnson, Annu. Rev. Fluid Mech. 37, 43 (2005).

[8] C. Verdant and D. De Backer, Curr. Opin. Crit. Care. 11, 240 (2005).

[9] C. M. Epstein, M. L. Evatt, A. Funk, L. Girard-Siqueira, N. Lupei, L. Slaughter, S. Athar, J. Green, W. McDonald, and M. R. DeLong, Clin. Neurophysiol. 118, 2189 (2007).

[10] M. De Mattei, A. Caruso, F. Pezzetti, A. Pellati, G. Stabellini, V. Sollazzo, and G. C. Traina, Connect. Tissue Res. 42, 269 (2001).

[11] S. S. Franklin, M. G. Larson, S. A. Khan, N. D. Wong, E. P. Leip, W. B. Kannel et al., Circulation 103, 1245 (2001).

[12] A. L. Pauca, M. F. O'Rourke, and N. D. Kon, Hypertension 38, 932 (2001).

[13] Y. Sano, Y. Kataoka, T. Ikuyama, M. Wada, H. Imano, K. Kawamura, T. Watanabe, A. Nishida, and H. Osanai, Bull. Phys. Fitness Res. Inst. 68, 17 (1988).

[14] K. Takazawa, N. Tanaka, M. Fujita, O. Matsuoka, T. Saiki, M. Aikawa, S. Tamura, and C. Ibukiyama, Hypertension 32, 365 (1998). 